\title{
The lichen flora of the Chagos Archipelago, including a comparison with other island and coastal tropical floras
}

\author{
Mark R.D.Seaward \\ Department of Environmental Science, University of Bradford, Bradford BD7 1DP, UK
}

\section{André Aptroot}

Centraalbureau voor Schimmelcultures, P.O.Box 273, 3740 AG Baarn, The Netherlands

\begin{abstract}
The 1996 Chagos Expedition provided the first opportunity to study the archipelago's lichen flora. Seventeen of the 55 islands were ecologically investigated, some in more detail than others, and lists and representative collections of lichens have been assembled for many of them. In all, 67 taxa have been recorded, 52 to specific level.

Although the islands have a low biodiversity for cryptogamic plants, as would be expected in terms of their relatively young age, remoteness and small terrestrial surface areas, those taxa that are present are often found in abundance and play significant ecological roles.

There is a good correlation between total lichen biodiversity and island size, despite the fact that Cocos nucifera is such an important substratum for cryptogamic plants and its presence on all islands studied provides a consistently high associated species count.

Comparisons of lichen floras for ten island and coastal tropical areas show good correlations (based on the Sörensen Coefficient) within the Indian Ocean as would be expected, but poorer correlations exist within and between Pacific Ocean and neotropical floras. Ranked correlations between Chagos and other floras are in the sequence Maldives > Laing Island > Aldabra > Tuamotu > Pitcairn $>$ N.Mariana \& Belize > Guadeloupe > Cook. When coefficients are calculated using only the Physciaceae, different correlations and sequences are derived, but the affinities of the Indian Ocean islands remain strong. However, although the lichen flora of Chagos is characteristic for an Indian Ocean, it is dominated by pantropical species.
\end{abstract}




\section{Introduction}

The Chagos Archipelago is the largest and most isolated atoll complex in the world. Athough the actual archipelago covers c. $37500 \mathrm{~km}$ of mainly coral reefs, only about $2 \%$ provides terrestrial habitats. The 55 islands of the archipelago (Figure 1) have a typical tropical maritime climate, with an annual rainfall of about $2400 \mathrm{~mm}$ and an average temperature of c. $27^{\circ} \mathrm{C}$. Recent research (Eisenhauer et al., 1999) has shown that the current islands vary in age between 2800 and 6500 years. For further information on the history, reef formation, climate, fauna and flora of these islands see Sheppard and Seaward (1999).

The islands were exploited for copra from the 1790s onwards; much of the native vegetation was removed to make way for Cocos plantations and many of the islands were inhabited by plantation workers. The plantations were run down in 1967 as their commercial future could not be guaranteed, and by 1973 the islands had become uninhabited, apart from the largest, Diego Garcia which supports a military base.

Diego Garcia $\left(44 \mathrm{~km}^{2}\right)$ is a horseshoeshaped atoll enclosing a large, deep lagoon with three small islands at its mouth. It was made available for British and USA defence purposes in 1966 and normally supports a military base of c. 3400 personnel. The other main island groups of the archipelago are the Peros Banhos atoll (29 islands, total land area of $10.4 \mathrm{~km}^{2}$ ) and the Salomon atoll (11 islands, total land area of 5.2 $\mathrm{km}^{2}$ ). The Great Chagos bank comprises eight islands widely separated from the other atolls. In addition, the Egmont atoll, formerly composed of six islands but now, as observed by the 1966 expedition members, evidently coalescing to form only three, is the smallest of the island groups.

Since their abandonment, most islands have been rarely visited, but extended visits by yachtsmen to the Salomon and Peros Banhos atolls are becoming increasingly common. The few other visitors have been mainly scientists, the most recent being the 1996 Chagos Expedition, permission for which centred on the requirement by the authorities for a conservation management plan, with much of the emphasis placed on an assessment of the status of the archipelago in biogeographical terms. Much of the research concentrated on the coral reefs and marine fauna, but two entomologists (Linda Barnett and Craig Emms) and three botanists (David Bellamy, Ursula Gregory and Mark Seaward) worked on terrestrial systems. Preliminary botanical work by Ursula Gregory on several islands of the Peros Benhos and Salomon atolls during early stages of the expedition was extended by more detailed studies, employing taxonomic, ecological and remote sensing techniques, made by the two other botanists who were fortunate in being able to visit a high proportion of the archipelago's islands.

\section{Ecology}

Despite their relatively young age and small terrestrial surface area, the islands have a relatively high biodiversity in terms of higher plants. Currently there are c. 280 species present, but only c. 35 of these comprise the original native flora prior to human arrival (Topp \& Sheppard, 1999). Since the cryptogamic plants had not previously been given serious attention, there being only a short published list of mosses (Townsend 1971), Mark Seaward (with supplementary work by Ursula Gregory and Fred Stewart) surveyed the lichen and bryophyte floras of many of the islands, and also made collections of cyanobacteria, algae and fungi. Although there is a relatively low diversity for most of these groups in terrestrial habitats, and any 'new' species discovered there will undoubtedly occur elsewhere, they are abundant and ecologically important.

Due to their small size and difficulty in identification, particularly in situ, more remain to be discovered, but to date only 9 moss, 4 liverwort, 68 lichen, 1 algal and 26 cyanobacterial taxa have been determined from strictly terrestrial habitats; a rich fungal flora undoubtedly exists, but due to the limited material available for study, only 21 macrofungal, 5 microfungal and 1 myxomycete taxa have been identified so far (Seaward 1999).

Lichens on Chagos would appear not to grow on coral, or on the poorly developed sandy soils arising from it, with the exception of an unnamed Siphula sp. which colonizes several square metres of exposed soil (pH c.7.4) on a single island. However, cyanobacteria, which extensively clothe eroded corals and sandy soils, 


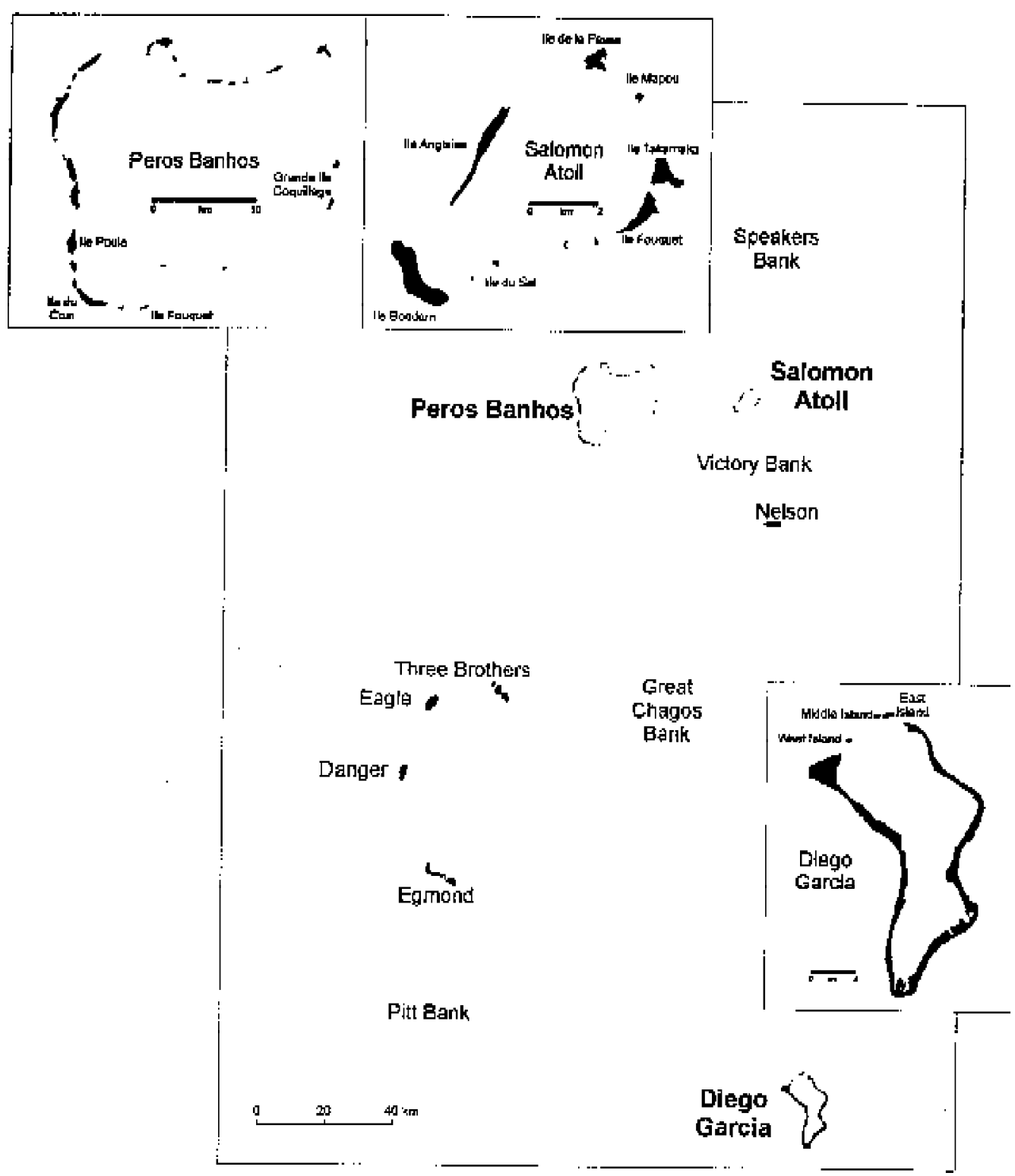

Fig. 1. Chagos Archipelago (see Table 1 for details of islands lichenologically studied). 
provide a continuously renewable nitrogen and carbon source and improve soil stability through particle aggregation. With time, such soils may support lichens, but in the meantime their role is upheld by the cyanobacterium Nostoc commune which covers very extensive areas of exposed soils, its presence only fully appreciated after rain.

However, bryophytes are important in stablising soils and appear to be the primary stages in the succession to higher vegetation in exposed areas of the islands. The soil around and below mosses is colonized by cyanobacteria which release not only substantial amounts of fixed nitrogen but also growth substances which benefit the moss (Rogers \& Henriksson 1976, Richardson 1981). The reduction or loss of breeding colonies of birds from islands due to rat infestation will lead to reduced inputs of phosphorus and nitrogen which will ultimately affect the capacity of some cryptogamic plants to establish themselves on soils inherently poor in nutrients.

Man-made building materials are similarly devoid of lichens, but cyanobacteria are to be found in abundance and relatively high diversity, their presence creating a characteristic black coating over the stonework, etc. of abandoned plantation buildings. Scytonema-Gloeocapsetum, the commonest association encountered in the Chagos islands, is well developed on such substrata where exposed to sunlight and wetted sporadically (Hoffmann 1989).

A rich cyanobacterial flora on the bark of many trees is supported by one or more as yet undetermined species of the alga Trentepohlia. Of the epiphytic floras studied to date, it would appear that Casuarina equisetifolia supports the largest diversity of cyanobacteria; Cocos nucifera, on the other hand, has only a few cyanobacteria species.

However, lichens readily colonize tree bark surfaces, particularly in the case of Cocos nucifera, its smooth, hard and usually dry bark supporting a rich lichen flora often dominating large areas of the trunk; however, there is a low diversity and poor coverage of bryophytes, except about the tree bases, particularly those subjected to higher humidity levels where cushions of the moss Calymperes tenerum can be found in plenty.
As Sipman and Harris (1989) note, lichens in tropical rain forests show little preference among phorophytes: this is amply demonstrated by the 20 or so phorophyte species studied on Chagos, with the exception of Cocos nucifera which due to its frequency appears to have developed its own distinctive epiphytic flora.

A lichen zonation from base to crown (cf. Sipman \& Harris 1989) is not distinguishable on Chagos trees, and although there are some differences between the epiphytic floras of the base, trunk and crown, zones are often delimited vertically according to aspect. In habitats frequented by birds, a natural lichen mosaic is often ill-defined due to the presence of other lichen species which are encouraged by the nutrient enrichment of tree trunks and branches. A 'nutrient rain' rich in leachates of excrement (Sipman \& Harris 1989) derived from tree canopy birds, their status and behaviour governed by the presence/absence of rats on particular islands, greatly influences the lichen flora.

The nature of the epiphytic flora changes with age, and as the trunks of all species die, different bryophytes support the decomposition process, the rotting wood community consisting mainly of Ectropothecium species (cf. Pocs 1982; Seaward 1999). Although a diversity of colourful fungi were also present, often forming a feature of the characteristic crater-shaped tree bases left when coconut palms topple over, lichens appeared not to be associated with this muscicolous habitat.

Two foliicolous lichens, Opegrapha vegae and Porina perminuta, are commonly found on Cocos leaves, which are also colonized by microfungi, such as Mycosphaerella gastonis; these become much more prevalent with time and a new microflora takes over once the leaves have been shed. The presence of foliicolous lichens on leaves other than those of Cocos and Calophyllum inophyllum was not investigated.

\section{Fieldwork}

Those terrestrial locations visited on the 1996 Expedition are shown in Figure 1 and listed in Table 1. In all, 25 islands were visited, but only 17 were investigated and collections, albeit variable 
Table 1. Islands of the Chagos Archipelago lichenologically studied during the 1996 Expedition

\begin{tabular}{|c|c|c|c|c|}
\hline Code & L ocation & $\begin{array}{l}\text { S i ze } \\
\text { (ha) }\end{array}$ & $\begin{array}{l}\text { L idhen } \\
\text { T axa* }\end{array}$ & Coll ectors** \\
\hline B & T hree B rothers: & & & \\
\hline $\mathrm{B}(\mathrm{N})$ & $\mathrm{N}$ arth & 6.0 & 11 & [1] \\
\hline$B(M)$ & M idde & 8.0 & 15 & [1] \\
\hline$B(S)$ & South & 23.0 & 17 & [1] \\
\hline DG & D iego G arcia & 2719.5 & 43 & [1] \\
\hline$D G(S)$ & D iego $G$ arcia (settlement) & & & [1] \\
\hline \multirow[t]{2}{*}{ DG( ) } & D iego G arcia (E ast, & & & \\
\hline & M idde \& West I slands) & 14.5 & & [3] \\
\hline$E$ & E agle I sland(s) & 243.5 & 20 & [1] \\
\hline EG & E gmont: & & & \\
\hline$E G(L)$ & L ubine & 177.0 & 18 & [1] \\
\hline E G (S) & Sipaille & 64.0 & 15 & [1] \\
\hline $\mathrm{N}$ & $\mathrm{N}$ eson $\quad \mathrm{I}$ sland & 81.0 & 13 & [1] \\
\hline \multirow[t]{4}{*}{ PB } & P eros $\quad B$ anhos: & & & \\
\hline & I le du Coin & 127.5 & & [2] \\
\hline & I le D iamante & 88.5 & & [2] \\
\hline & I leY eye & 58.5 & & [2] \\
\hline 9 & Sal omon I slands: & & & \\
\hline$S I(A)$ & I le A nglaise & 75.5 & 15 & {$[1,2]$} \\
\hline SI (B ) & I le B oddam & 108.5 & 32 & {$[1,2]$} \\
\hline SI (D) & I le Diable & 0.1 & 8 & [1] \\
\hline SI (DP) & I le de la Passe & 26.0 & 10 & {$[1,2]$} \\
\hline SI (DS) & I le du Sel & 2.0 & & {$[2]$} \\
\hline SI (F) & I le Fouquet & 39.5 & 21 & {$[1,2]$} \\
\hline SI ( ) & I le J acobin & 1.5 & 8 & {$[1,2]$} \\
\hline SI (M) & I le M apou & 4.0 & 10 & {$[1,2]$} \\
\hline SI (P) & I le P oule & 0.5 & & {$[2]$} \\
\hline SI (S) & I le Sepulture & 0.5 & 14 & [1] \\
\hline SI (T) & I le T akamaka & 44.0 & 15 & {$[1,2]$} \\
\hline * M ini & imal no. of taxa determined. & & & \\
\hline
\end{tabular}

in size and diversity, made of different groups of cryptogamic plants. Collections from East, Middle and West Islands of Diego Garcia, all islands of Peros Banhos, and Ile Poule and Ile du Sel of the Salomon Islands are limited in scope, the author having been unable to visit these (as well as Danger Island, Sea Cow and Resurgent) in order to make field notes and collect representative material.

Based on the fieldwork undertaken during the 1996 Expedition, the lichen flora list which follows is almost complete, and an attempt has been made to determine the status of the taxa for each of the islands and their relationship to (1) neighbouring islands within the archipelago, (2) other islands in the Indian Ocean, and (3) to other island and coastal tropical floras of the world. Relationships between the Chagos lichen flora and those of the mainlands of Africa, India and South-east Asia have yet to be determined (cf. 
Table 2. Relationships between ten island and coastal lichen floras based on Sorensen coefficients (K) for (a) all taxa and (b) only Physciaceae. Sources: Aldabra - Seaward et al. (1996); Belize - Barclay-Estrup (1992); Chagos - Seaward (1999); Guadeloupe - Aptroot (1998, unpublished list); Laing Island - Aptroot et al. (1995); Maldives - Aptroot (1991); Cook, N.Mariana, Pitcairn \& Tuamotu Elix \& McCarthy (1998).

Contrasting L ocations

$G$ ood correlation:

$M$ al dives A Idabra

$C$ hagos $\quad M$ aldives

$R$ easonable correlation:

T uamotu P itcairn

$C$ hagos $L$ aing I $S$

N.M ariana C ook

C hagos A I dabra

A Idabra T uamotu

N.M ariana P itcairn

Chagos T uamotu

N.M ariana T uamotu

$M$ aldives $T$ uamotu

$C$ hagos $P$ itcairn

$M$ aldives $L$ aing I $S$

M oderate correlation:

$M$ aldives $G$ uadel oupe

C ook P itcairn

$M$ al dives $\quad P$ itcairn

$M$ aldives $B$ el ize

Chagos N.M ariana

$C$ hagos

$M$ al dives

A I dabra

C hagos

$T$ uamotu

$P$ itcairn

A I dabra

$\mathrm{L}$ aing I $\mathrm{S}$
$B$ elize

C ook

G uadel oupe

$G$ uadel oupe

$B$ el ize

B elize

$P$ itcairn

G uadel oupe (a)

K R ank

$34.5 \quad 1$

32.82

24.53

24.14

23.35

$22.6 \quad 6$

22.27

$22.0 \quad 8$

21.69

$19.8 \quad 10$

$19.5 \quad 11$

$18.7 \quad 12$

$18.7 \quad 12$

$\begin{array}{ll}15.1 & 14\end{array}$

$14.8 \quad 15$

14.316

14.316

13.118

13.118

$13.0 \quad 20$

$12.5 \quad 21$

11.622

$11.4 \quad 23$

11.124

$10.8 \quad 25$

10.626 (b)

$\mathrm{R}$ ank

W eak correlation:

$\begin{array}{ll}\text { A I dabra } & \text { C ook } \\ \text { L aing I S } & \text { T uamotu } \\ \text { A I dabra } & \text { N.M ariana } \\ \text { L aing I S } & \text { N.M ariana } \\ \text { A I dabra } & \text { B el i ze } \\ \text { A I dabra } & \text { L aing I s } \\ \text { G uadel oupe } & \text { B el ize } \\ \text { C hagos } & \text { C ook } \\ \text { T uamotu } & \text { C ook } \\ \text { L aing I S } & \text { B el ize } \\ \text { M al di ves } & \text { N.M ariana }\end{array}$

$9.8 \quad 27$

18

$\begin{array}{ll}9.8 & 27\end{array}$

14

$8.7 \quad 29$

$8.7 \quad 29$

$8.7 \quad 29$

8.632

8.533

$7.6 \quad 34$

7.535

$5.8 \quad 36$

$5.4 \quad 37$ 
P oor correlation:

\begin{tabular}{|c|c|c|c|c|}
\hline $\mathrm{L}$ aing I $\mathrm{S}$ & P itcairn & 4. & 38 & 24 \\
\hline C ook & G uadel oupe & 3. & 39 & $37 *$ \\
\hline N .M ariana & B el ize & 2. & 40 & 29 \\
\hline $\mathrm{L}$ aing I s & C ook & 2. & 41 & $37 *$ \\
\hline N.M ariana & G uadel oupe & 2. & 42 & $37 *$ \\
\hline \multicolumn{5}{|c|}{ N 0 correlation: } \\
\hline T uamotu & G uadel oupe & 0 & 43 & $37 *$ \\
\hline P itcairn & G uadel oupe & 0 & 43 & 37 \\
\hline C ook & B elize & 0 & 43 & $37 *$ \\
\hline
\end{tabular}

Renvoize 1975).

The relationship between biodiversity and island size has being investigated: preliminary studies show that there is a good correlation $(\mathrm{r}=$ 0.7465 ) between the total number of lichen taxa and island size (Figure 2), despite the fact that Cocos is such an important substratum for lichens and its presence on all the islands studied provides a consistently high associated species count.

As a result of the 1996 Expedition, it was hoped to establish credible baseline data for future studies aimed at monitoring lichen stability in the light not only of local disturbance but also far-ranging global impacts. Although this may be possible for the islands' terrestrial habitats, it should be noted that lichens did not occur on exposed coral of the island fringes: zonal patterns capable of providing accurate monitors of seawater pollution and of rising sea-levels were lacking. The algal and cyanobacterial floras of the marine and tidal areas of the islands, which are undoubtedly much more biodiverse and contain many native, and indeed endemic, species, may be better suited to monitor such changes.

\section{List of Taxa Recorded}

Sixty-seven taxa were recorded, 52 and 15 determined to specific and generic level. The taxonomic list provides in the following sequence (1) some indication of frequency of occurrence; (2) preferred substrata; (3) distribution in the
Chagos Archipelago (according to the abbreviations given under 'Locations Investigated' above); and (4) world distribution where known. Specimens of all the taxa recorded by Mark Seaward are in Herb.Seaward, and in the case of collections made by Ursula Gregory in Herb.E; duplicates of some material collected by the author have been deposited in Herb. Aptroot, B, BG, BM, E, Ekman, H, IMI, Lücking, MO and OXF.

Agonimia papillata (O.Eriksson) Diederich \& Aptroot Occasional, on Hernandia sonora \& bases of Pisonia grandis.

B(M); B(S). Pantropical.

Herb. Aptroot, OXF, MRDS 107322, 107506

Anisomeridium subprostans (Nyl.) R.C.Harris

Rare, on Hernandia sonora.

SI(F). Pantropical.

Herb. MRDS 107418

Arthonia antillarum (Fée) Nyl.

Locally frequent on Scaevola sericea; less frequent on Argusia argentea, Cocos nucifera, Guettarda speciosa \& Hernandia sonora. $\mathrm{B}(\mathrm{M}) ; \mathrm{B}(\mathrm{N}) ; \mathrm{B}(\mathrm{S}) ; \mathrm{DG} ; \mathrm{DG}(\mathrm{I}) ; \mathrm{EG}(\mathrm{L}) ; \mathrm{EG}(\mathrm{S})$; N; SI(DP). Pantropical.

Herb. Aptroot, MRDS 107371, 107399 , 107430, 107434

Arthonia sp.A

[cf. A.ravida Stirton] 
Rare, on Cocos nucifera.

$\mathrm{B}(\mathrm{S})$.

Herb. MRDS 108367

Arthonia sp.B nucifera. on Casuarina equisetifolia \& Cocos DG;EG(L).

Herb. IMI 374554

Arthopyrenia majuscula (Nyl.) Zahlbr.

Locally frequent, particularly on Scaevola sericea; less frequent on Barringtonia speciosa, Casuarina equisetifolia, Cocos nucifera, Guettarda speciosa \& Premna obtusifolia. $\mathrm{B}(\mathrm{M}) ; \mathrm{B}(\mathrm{N}) ; \mathrm{B}(\mathrm{S}) ; \mathrm{DG} ; \mathrm{E} ; \mathrm{EG}(\mathrm{L}) ; \mathrm{EG}(\mathrm{S}) ; \mathrm{N}$;

SI(A); SI(B); SI(D); SI(F). Pantropical.

Herb. Aptroot, E, OXF, MRDS 107325 , 107326, 107354, 107355, 107398, 107427, 107508, 107513

Bacidia hostheleoides (Nyl.) Zahlbr.

Frequent, on Argusia argentea, Cocos nucifera, Guettarda speciosa, Hernandia sonora, Scaevola sericea \& lignum.

B(M); DG; DG(I); E; EG(L); EG(S); N; SI(A); SI(B); SI(F); SI(J); SI(S); SI(T). Neotropical. Herb. BM, MRDS 108120, 108121, 108122, 108805

Bacidia medialis (Nyl.) Zahlbr.

Rare, on Argusia argentea \& lignum. DG, DG(I), N, SI(J). Pantropical. Herb. MRDS 108123, 108800, 108880

Bacidia sp.

On Argusia argentea, Cocos nucifera \& Hernandia sonora.

B(N); DG; DG(I); EG(L); N; SI(S); SI(T).

Herb. MRDS 108801, 108802

Buellia efflorescens Müll.Arg.

Common on Argusia argentea, Cocos nucifera \& Morinda citrifolia.

$\mathrm{B}(\mathrm{M}) ; \mathrm{B}(\mathrm{N}) ; \mathrm{B}(\mathrm{S}) ; \mathrm{DG}$; DG(I); E; EG(L); EG(S); N; SI(A); SI(B); SI(F); SI(S). Pantropical.

Herb. MRDS 107352, 107353, 107364, $107375,107502,107575,107581$
Byssolecania fumosonigricans (Müll.Arg.) R.Sant.

Rare, on green stem of unidentified plant. DG. Pantropical.

Herb. MRDS 108881

Calicium hyperelloides Nyl.

Rare, on Cocos nucifera.

SI(B). Pantropical to subtropical.

Herb. MRDS 107323

\section{Caloplacasp.}

Rare, on Casuarina equisetifolia \& Scaevola sericea.

DG;EG(L);EG(S).

Herb. MRDS 108803

\section{Chrysothrix candelaris (L.) Laundon}

Uncommon, on Casuarina equisetifolia. DG; SI(B). Cosmopolitan.

Herb. Aptroot, B, BG, E, OXF, MRDS 107340, 107392, 107540, 107545

\section{Coccocarpia cf. domingensis Vainio}

Rare, on Cocos nucifera.

SI(B). Pantropical.

Herb. MRDS 107351

\section{Coccocarpia erythroxyli (Sprengel) Swinscow \&}

Krog

Rare, on Cocos nucifera.

SI(B). Pantropical to subtropical.

Herb. MRDS 107383

\section{Coccocarpia palmicola (Sprengel) Arvidsson \&} Galloway

Locally frequent on Cocos nucifera, Casuarina equisetifolia \& Terminalia catappa. DG;E;EG(L); SI(B); SI(DS). Pantropical. Herb. Aptroot, B, BM, E, OXF, MRDS 107321, 107324, 107339, 107391, 107421, 107515, 107576,108068

\section{Coccocarpia rottleri (Ach.) Arvidsson}

Rare, on Cocos nucifera.

SI(B). Pantropical.

Herb. MRDS 107621

\section{Coenogonium sp.}

Locally frequent on stumps, adventitious 
roots \& husks of Cocos nucifera; apothecia infrequent.

$$
\text { B(M); DG; E; N; SI(B); SI(J). }
$$

\section{Collema rugosum Krempelh.}

Locally frequent on Artrocarpus altilis, Barringtonia speciosa, Calophyllum inophyllum, Casuarina equisetifolia, Cocos nucifera, Cordia subcordata, Hernandia sonora, Hibiscus tiliaceus, Intsia bijuga, Morinda citrifolia, Premna obtusifolia \& Terminalia catappa.

DG; DG(S); SI(A); SI(B); SI(D); SI(DS); $\mathrm{SI}(\mathrm{F}) ; \mathrm{SI}(\mathrm{S}) ; \mathrm{SI}(\mathrm{T}) \quad$ - probably on all islands. Palaeotropical.

Herb. MRDS 107318, 107319, 107348, 107373, 108109

\section{Cryptolechia subincolorella (Nyl.) D.Hawksw.}

Infrequent, on Hernandia sonora, Intsia bijuga, Morinda citrifolia, Pisonia grandis \& Tabebuia pallida.

B(S); DG; E; SI(B); SI(F); SI(S); SI(T). Paleotropical.

Herb. OXF, MRDS 107317, 107349, 107350, 107388

\section{Dimerella sp.}

Rare, on adventitious roots of Cocos nucifera \& lignum.

DG; SI (J).

Herb. MRDS 108804

\section{Dirinaria applanata (Fée) Awasthi}

Infrequent, on Barringtonia speciosa, Casuarina equisetifolia \& Cocos nucifera.

DG; SI(F); SI(S). Pantropical.

Herb. MRDS 107374, 107514

\section{Dirinaria picta (Sw.) Clem.\& Shear}

Common, on Artrocarpus altilis, Barringtonia speciosa, Calophyllum inophyllum, Casuarina equisetifolia, Cocos nucifera, Intsia bijuga \& Scaevola sericea. B(S); DG; E; EG(L); EG(S); SI(A); SI(B); SI(D); SI(DP); SI(F); SI(M); SI(S); SI(T) - probably on all islands. Pantropical.

Herb. Aptroot, E, OXF, MRDS 107330, 107333, 107335, 107336, 107338, 107342, 107393, 107405, 107420, 107423, 107424,
107500, 107505, 107559

Endocarpon pusillum var. pallidum (Ach.) Körber Rare, on cementwork of wall-tops, abandoned plantation buildings and stone memorials. DG; SI(B). Cosmopolitan. Herb. MRDS 107312, 107313

Fellhanera subtilis (Vezda) Diederich \& Sérusiaux

Rare, on ? Cocos nucifera.

DG; DG(I).

Widespread (mainly European, but probably cosmopolitan).

Herb. MRDS 107737

\section{Graphina analoga (Nyl.) Zahlbr.} Uncommon, on Cocos nucifera \& Terminalia catappa.

SI(B); SI(F). Pantropical.

Herb. MRDS 107345, 107346

\section{Graphina streblocarpa (Bel.) Müll.Arg.} Infrequent, on Cocos nucifera (particularly about base on adventitious roots) \& Melifera indica. DG; SI(B). Pantropical. Herb. OXF, MRDS 107344, 107347

\section{Graphis lineola Ach.} Infrequent, on Barringtonia speciosa \& Casuarina equisetifolia. DG; SI(F). Pantropical. Herb. OXF, MRDS 107554, 107561, 108366

Graphis sp(p). on Barringtonia speciosa(?) \& Casuarina equisetifolia. DG; DG(S); EG(S); SI(B); SI(DP).

\section{Hyperphyscia syncolla (Nyl.) Kalb} Rare, on Cocos nucifera. B(M). Pantropical. Herb. MRDS 107435

\section{Lecanora achroa Nyl.}

Occasional on Barringtonia speciosa, Casuarina equisetifolia \& Cocos nucifera. DG; SI(B); SI(F). Pantropical. 

107796

Herb. E, OXF, MRDS 107794, 107795 ,

Lecanora ecoronata Vainio

Occasional on Guettarda speciosa \&

Scaevola sericea.

$\mathrm{B}(\mathrm{S}) ; \mathrm{E} ; \mathrm{EG}(\mathrm{L}) ; \mathrm{EG}(\mathrm{S}) ; \mathrm{SI}(\mathrm{B}) ; \mathrm{SI}(\mathrm{F})$.

Probably pantropical.

Herb. MRDS 107792, 107793

\section{Lecanora sp.}

[cf. L.expallens Ach.]

Frequent, on Argusia argentea,

Casuarina equisetifolia, Cocos nucifera \& Scaevola sericea.

SI(DS); SI(T).

DG; DG(I); EG(L); EG(S); SI(A); SI(B);

Herb. E, MRDS 107881, 107882, 107885

Lecanora leproplaca Zahlbr.

Frequent, on Cocos nucifera, Cordia subcordata \& Premnea obtusifolia

DG; E; EG(L); SI(A); SI(B); SI(J).

Pantropical.

Herb. MRDS 107797, 107798

\section{Lecanora tropica Zahlbr.}

Rare, on Cocos nucifera.

E. Pantropical.

Herb. MRDS 107791

\section{Lecanora sp.}

Perhaps an undescribed sp. [cf.

L.usambarensis Müll.Arg.]

Rare, on Casuarina equisetifolia

DG.

Herb. MRDS 107883

\section{Lithothelium hyalosporum (Nyl.) Aptroot}

Uncommon, on Cordia subcordata \&

Hernandia sonora.

SI(F); SI(M). Cosmopolitan.

Herb. MRDS 107368, 107417

\section{Lithothelium illotum (Nyl.) Aptroot}

Rare, on Intsia bijuga.

SI(T). Pantropical.

Herb. MRDS 107620
Nadvornikia hawaiense (Tuck.) Tibell

Rare, on Cocos nucifera.

DG. Pantropical.

Herb. Aptroot, OXF, MRDS 107377, 107378

\section{Opegrapha vegae R.Sant.}

Common, on Cocos nucifera leaves.

$\mathrm{B}(\mathrm{M}) ; \mathrm{B}(\mathrm{N}) ; \mathrm{B}(\mathrm{S}) ; \mathrm{DG} ; \mathrm{EG}(\mathrm{L}) ; \mathrm{EG}(\mathrm{S}) ; \mathrm{N}$; SI(A); SI(B); SI(DP); SI(F); SI(S); SI(T) probably on all islands. Pantropical.

Herb. Aptroot, B, BG, BM, E, Lücking, OXF, MRDS 107307, 107308, 107309, 107310, 107311, 107412, 107413, 107414, 107415

\section{Opegraphasp.A}

[cf. O.confertoides Torrente \& Egea]

Rare, on Cocos nucifera.

SI(DP).

Herb. MRDS 108341

\section{Opegraphasp.B}

[cf. O.rufescens Pers.]

Uncommon, on Argusia argentea, Cocos nucifera \& Herandiasonora.

$\mathrm{B}(\mathrm{M}) ; \mathrm{B}(\mathrm{N}) ; \mathrm{B}(\mathrm{S}) ; \mathrm{N}$.

Herb. MRDS 108338, 108340, 108342, 108345,108355

\section{Opegraphasp.C}

[cf. O.viridis (Ach.) Nyl.]

Common, on Argusia argentea, Calophyllum inophyllum, Casuarina equisetifolia, Cocos nucifera, Cordia subcordata, Hernandia sonora, Hibiscus tiliaceus, Intsia bijuga, Morinda citrifolia, Pisonia grandis \& Tabebuia pallida; on all islands studied.

Herb. Aptroot, E, MRDS 108339, 108343, 108344, 108346, 108349, 108350, 108351, 108352, 108356, 108357, 108359

\section{Opegraphasp.D}

[cf. O.vulgata (Ach.) Ach.]

Rare, on Cocos nucifera \& Scaevola sericea.

SI(D); SI(DP).

Herb. MRDS 108347, 108358

\section{Opegraphasp.E}

Infrequent, on Argusia argentea, Calophyllum inophyllum, Casuarina equi- 
setifolia, Cocos nucifera, Cordia subcordata,

Hernandia sonora, Hibiscus tiliaceus \& Intsia bijuga.

B(M); DG; DG(I); E; EG(L); EG(S); SI(A);

SI(B); SI(F); SI(M); SI(T).

Herb. MRDS 108347, 108358

Phaeographis sericea (Eschw.) Müll.Arg.

Uncommon, on Casuarina equisetifolia.

DG. Pantropical.

Herb. MRDS 108799

\section{Physcia cf. dimidiata (Arnold) Nyl.}

Rare, substratum unidentified.

SI(D). Cosmopolitan.

\section{Physcia erumpens Moberg}

Uncommon, on Cocos nucifera \& Hernandia sonora, often over a cushion of moss (Calymperes spp.).

DG - ? restricted to one island. Pantropical. Herb. MRDS 107342, 107343

\section{Physcia integrata Nyl.}

Locally frequent on Cocos nucifera; less common on Guettarda speciosa \& Hernandia sonora.

$\mathrm{B}(\mathrm{M}) ; \mathrm{B}(\mathrm{N}) ; \mathrm{B}(\mathrm{S})$ - ? restricted to The Three Brothers. Pantropical.

Herb. Aptroot, OXF, MRDS 107384, 107403, 107432, 107517

\section{Physcia krogiae Moberg}

Rare, on Pisonia grandis.

$\mathrm{B}(\mathrm{S})$. Widespread in tropical \& subtropical areas.

Herb. OXF, MRDS 107358

\section{Physcia sorediosa (Vainio) Lynge}

Common on Calophyllum inophyllum, Casuarina equisetifolia, Cocos nucifera, Cordia subcordata, Guettarda speciosa, Hernandia sonora, Intsia bijuga \& Scaevola sericea; uncommon on cement \& rare on ironwork.

DG; E; EG(L); EG(S); SI(A); SI(B); SI(D); SI(DP); SI(DS); SI(F); SI(J); SI(M); SI(S); SI(T) probably on all islands. Pantropical.

Herb. Aptroot, B, BM, E, H, OXF, MRDS 107327, 107328, 107334, 107341, 107356, 107357,
107367, 107370, 107389, 107394, 107395, 107396 107397, 107406, 107416, 107419, 107499, 107501, 107503,107560

\section{Porina perminuta Vainio}

Common on Cocos nucifera leaves; locally frequent on Calophyllum inophyllum leaves.

$\mathrm{B}(\mathrm{M}) ; \mathrm{B}(\mathrm{N}) ; \mathrm{DG} ; \mathrm{E} ; \mathrm{EG}(\mathrm{L}) ; \mathrm{EG}(\mathrm{S}) ; \mathrm{N} ; \mathrm{SI}(\mathrm{A})$; $\mathrm{SI}(\mathrm{B}) ; \mathrm{SI}(\mathrm{F}) ; \mathrm{SI}(\mathrm{J}) ; \mathrm{SI}(\mathrm{S})$ - probably on all islands. Mainly Pacific islands.

Herb. Aptroot, B, BG, BM, E, H, Lücking, OXF, MRDS 107301, 107302, 107303, 107304, 107305, 107306, 107407, 107408, 107409, 107410, 107411,107757

\section{Pyrenula concatervans (Nyl.) R.C.Harris}

Locally frequent on Calophyllum inophyllum, Guettarda speciosa, Hibiscus tiliaceus, Pisonia grandis \& Tabebuia

pallida; frequent on Intsia bijuga. B(S); DG; E; SI(A); SI(B); SI(DS); SI(F); SI(S); SI(T). Pantropical. Herb. Aptroot, E, MRDS 107362, 107365 , 107366, 107369, 107385, 107558

\section{Pyrenula confinis (Nyl.) R.C.Harris}

Uncommon, on Casuarina equisetifolia \& Premna obtusifolia.

DG. Pantropical.

Herb. MRDS 107390, 107504, 107516, 107556

\section{Pyrenula macularis (Zahlbr.) R.C.Harris} Uncommon, on Casuarina equisetifolia \& Intsia bijuga.

DG; SI(T). Pantropical.

Herb. MRDS 107361, 107510

\section{Pyrenula nitidula (Bres.) R.C.Harris}

Rare, on Hernandia sonora. DG. Pantropical.

Herb. OXF, MRDS 107431

\section{Pyrenula ochraceoflava (Nyl.) R.C.Harris}

Abundant on Cocos nucifera; less common on Barringtonia speciosa, Calophyllum inophyllum, Casuarina equisetifolia, Hernandia catappa, Pisonia grandis, Premna obtusifolia \& Scaevola sericea. 
$\mathrm{B}(\mathrm{M}) ; \mathrm{B}(\mathrm{N}) ; \mathrm{B}(\mathrm{S}) ; \mathrm{DG} ; \mathrm{E} ; \mathrm{EG}(\mathrm{L}) ; \mathrm{EG}(\mathrm{S}) ; \mathrm{N}$; SI(A); SI(B); SI(D); SI(DP); SI(F); SI(M); SI(S); SI(T). On all islands studied. Pantropical.

Herb. Aptroot, B, BG, BM, E, OXF, MRDS 107314, 107315, 107337, 107360, 107376, 107422, 107428, 107429, 107436, 107498, 107538, 107546

Pyrenula parvinuclea (Meyen \& Fw.) Aptroot Uncommon, on Barringtonia speciosa, Casuarina equisetifolia, Cocos nucifera, Guettarda speciosa \& Scaevola sericea.

B(S); DG; E; SI(F). Pantropical.

Herb. OXF, MRDS 107359, 107400, 107555

Pyxine cocoes (Sw.) Nyl.

Common, on Argusia argentea, Cocos

nucifera, Cordia subcordata, Guettarda speciosa \& Hernandia sonora.

$\mathrm{B}(\mathrm{M}) ; \mathrm{B}(\mathrm{N}) ; \mathrm{B}(\mathrm{S})$; DG; DG(I); E; N; SI(A); SI(B); SI(M); SI(S); SI(T). Cosmopolitan.

Herb. MRDS 107329, 107331, 107332, 107363, 107386, 107387, 107433, 107512, 107582

\section{Pyxine consocians Vainio}

Locally frequent, on Cocos nucifera. DG; E; SI(DP); SI(B); SI(M). Pantropical. Herb. OXF, MRDS 107372, 107511, 108798

\section{Pyxine reticulata (Vainio) Vainio}

Rare, on Cocos nucifera.

E. Pantropical.

Herb. MRDS 107402

\section{Pyxine retirugella Nyl.}

Rare, substratum unidentified. DG; SI(M). Pantropical.

\section{Rinodina sp.}

Rare, on Cocos nucifera.

DG.

\section{Siphula sp.}

Rare, on thin poorly developed soil, covering several square metres.

$\mathrm{SI}(\mathrm{B})$.

Herb. Aptroot, MRDS 108110

Thelenella modesta (Nyl.) Nyl.

Rare, on lignum.

SI(M). Cosmopolitan.
Herb. E, MRDS 108229

Tomasellia eschweileri (Müll.Arg.) R.C.Harris Rare, but overlooked, on Cocos nucifera. SI(B). Pantropical. Herb. MRDS 107404

Verrucaria cf. maculiformis Krempelh.

Rare, on gravestone in abandoned plantation village.

DG. Cosmopolitan.

\section{Geographical Affinities of the Flora}

As can be seen from above, the lichen flora of Chagos mainly comprises pantropical species. The flora is characteristic of coastal tropical environments, and highly comparable to other small oceanic islands because of the absence of mountain species and species restricted to dense rainforest. The Chagos flora mainly consists of crustose lichens, except for the Physciaceae (syn. Pyxinaceae). As knowledge of tropical crustose lichens is still very incomplete, it is difficult to compare total species lists between different areas as the method of sampling and identification varies.

Nevertheless, a comparison can be made with some smaller islands which have been as exhaustively sampled by the authors as Chagos, namely the Maldives in the Indian Ocean (Aptroot 1991) and Laing Island in the Pacific Ocean (Aptroot et al. 1995). Less complete species lists are available for most smaller island groups in the Pacific Ocean (Elix \& McCarthy 1998) and for Aldabra in the Indian Ocean (Seaward et al. 1996). For further comparisons, emphasis has been laid on some dominant elements in the Chagos flora, ie. Physciaceae and species growing on Cocos. Furthermore, comparisons could be made with neotropical sites, but no suitable species lists have been published in recent years. Therefore, a comparison is made with the epiphytic flora on Cocos in Belize (Barclay-Estrup 1992) and with unpublished 1998 records from Guadeloupe (Aptroot, unpublished data).

Relationships between ten different island and coastal tropical floras were tested by means 


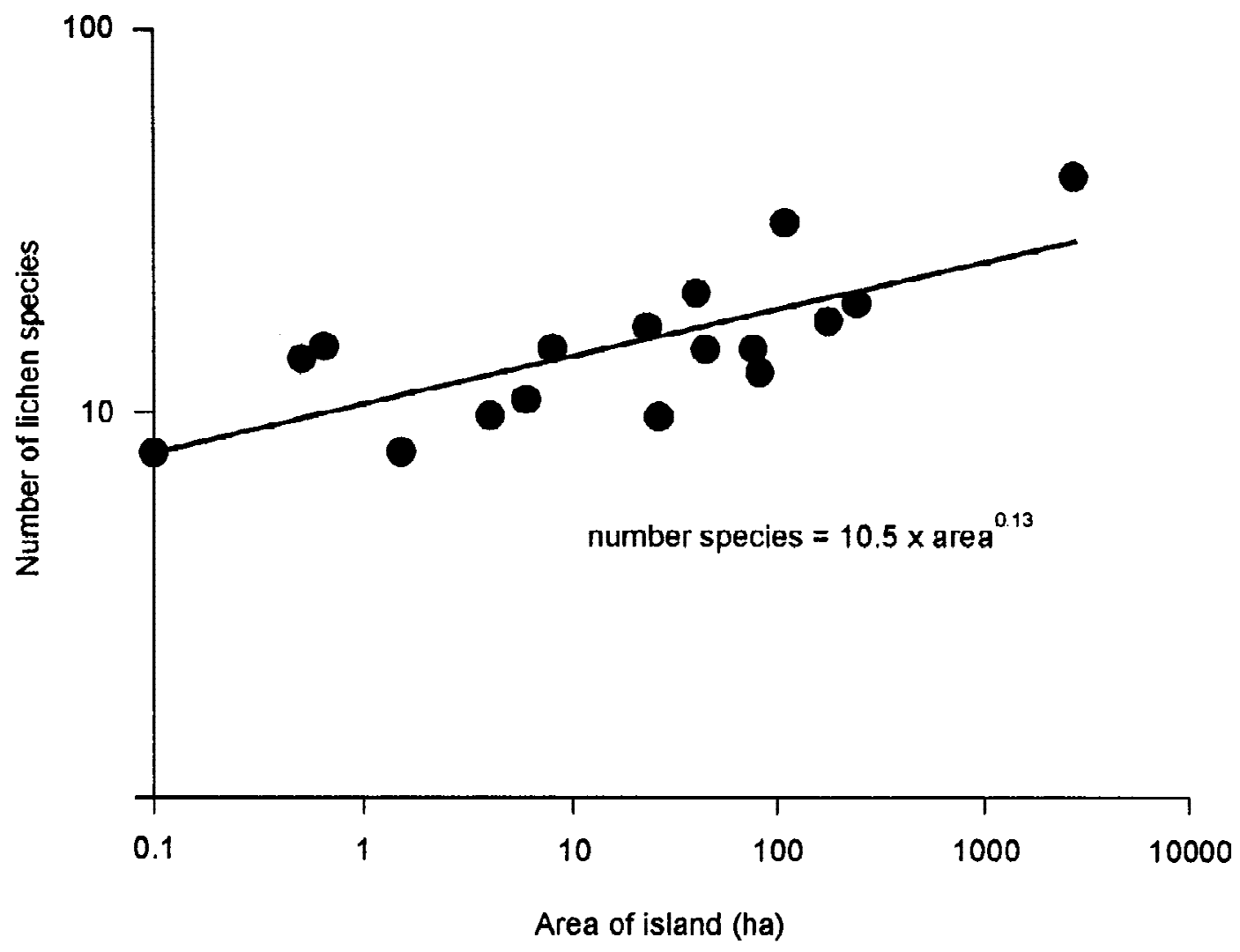

size on the number of lichen taxa (see also Table 1).

of the Sörensen Coefficient (K):

$$
K=\frac{200 \times c}{a+b}
$$

where $\mathrm{a}=$ number of species in one region, $\mathrm{b}=$ number of species in another region, and $\mathrm{c}=$ number of species common to both regions. As can be seen from Table 2, good correlations of lichen floras occur within the Indian Ocean, as would be expected, but poorer correlations existed within and between other geographical regions. Surprisingly, in the case of Chagos, its lichen flora showed an interesting ranking with other floras; in view of the predominating pantropical element, an overall more or less equal similarity between the floras was expected. Wide-ranging ranked correlations between Chagos and other floras are in the sequence Maldives > Laing Island > Aldabra > Tuamotu > Pitcairn > N.Mariana \& Belize > Guadeloupe > Cook. Although comparison of the Physciaceae of the ten floras (Table 2) show similarly high correlations between the Indian Ocean islands, the overall ranking is completely different. Comparisons with the Physciaceae floras of Madagascar (Aptroot 1988), Colombia (Aptroot 1989) and the Guianas (Aptroot 1987) have yet to be undertaken.

It is therefore concluded that although the lichen flora of Chagos is characteristic for an Indian Ocean island, it is dominated by pantropical species.

\section{Acknowledgements}

The senior author (MRDS) is particularly grateful to The Royal Society, British Ecological Society, The 
Percy Sladen Memorial Fund, The Colin Reid Countryside Trust and the University of Bradford for funding which allowed him to participate in the 1996 Chagos Expedition; he also acknowledges with thanks the support of expedition members, particularly Prof.D.Bellamy, Dr C.R.C. Sheppard, Mr J.M.W. Topp, Cmdr N. Wells, Miss U. Gregory and Dr F. Stewart. Thanks are also due to Dr J. Egea (Opegrapha), Dr S. Ekman (Bacidia), Dr T.Lumbsch (Lecanora), Dr E. Serusiaux (Fellhanera) and Dr H.J.M. Sipman (foliicolous lichens) for identifying or confirming critical taxa as indicated, and to Mr S. Davidson and Dr A.D. Headley for their preparation of Figures 1 and 2 respectively.

\section{References}

Aptroot,A. 1987. Pyxinaceae (Lichens). Flora of the Guianas, series E, 1: 1-59.

Aptroot,A. 1988. Lichens of Madagascar: the Pyxinaceae (syn. Physciaceae).Cryptogamie, Bryol.Lichenol. 9: 141-147.

Aptroot,A. 1989. Studies on Colombian cryptogams. XL. The family Pyxinaceae (Lichenized Fungi). Proceedings of the Koninklijke Nederlandse Akademie van Wetenschappen, series C, 92: 269-280.

Aptroot,A. 1991. The lichen flora of The Maldives. Lichenologist 23: 57-60.

Aptroot,A., Diederich,P., Serusiaux,E. \& Sipman,H. 1995. Lichens and lichenicolous fungi of Laing Island (Papua New Guinea). Bibliotheca Lichenologica 57: 19-48.

Barclay-Estrup,P. 1992. Lichens of Cocos nucifera on Caye Caulker, Belize. Lichenologist 24: 249-254.

David,J.C. \& Hawksworth,D.L. 1995. Lichens of Mauritius I: some new species and records. Bibliotheca Lichenologica 57: 93-111.

Eisenhauer,A., Heiss,G.A., Sheppard,C.R.C. \& C.Dullo,W.-C. 1999. Reef and island formation and Late Holocene sea-level changes in the Chagos islands. In:Ecology of the Chagos Archipelago(ed. C.R.C. Sheppard \& M.R.D.Seaward): 21-33. Westbury Press/Linnean Society, London.

Elix,J.A. \& McCarthy,P.M. 1998. Catalogue of the lichens of the smaller Pacific islands. Bibliotheca Lichenologica 70: 1-361.

Hoffmann,L. 1989. Algae of terrestrial habitats. Botanical Reviews 55: 77-105.

Pocs,T. 1982. Tropical forest bryophytes. In: Bryophyte Ecology (ed. A.J.E.Smith): 59-104. Chapman \& Hall, London.

Renvoize,S.A. 1975. A floristic analysis of the western Indian Ocean coral islands. Kew Bulletin 30: 133-152.

Richardson,D.H.S. 1981. The Biology of Mosses . Blackwell, Oxford.

Rogers,G.A. \& Henriksson,E. 1976. Associations between the blue-green algae Anabaena variabilis and Nostoc muscorum and the moss Funaria hygrometrica with reference to the colonization of Surtsey. Acta Botanica Islandica 4: 10-15.

Seaward,M.R.D. 1999. Cryptogamic flora of the Chagos Archipelago.In: Ecology of the Chagos Archipelago (ed. C.R.C.Sheppard \& M.R.D.Seaward): 207-224. Westbury Press/Linnean Society, London.

Seaward,M.R.D., Hambler,C. \& Aptroot,A. 1996. Bryophytes and lichens of Aldabra.Tropical Bryology 12: 29-33.

Sheppard,C.R.C. \& Seaward,M.R.D.,ed. 1999. Ecology of the Chagos Archipelago. Westbury Press/Linnean Society, London.

Sipman,H.J.M. \& Harris,R.C. 1989. Lichens. In: Tropical Rain Forest Ecosystems (ed. H.Lieth \& M.J.A.Werger): 303-309. Elsevier, Amsterdam.

Topp,J.M.W. \& Sheppard,C.R.C. 1999.Higher plants of the Chagos Archipelago. In: Ecology of the Chagos Archipelago (ed. C.R.C.Sheppard \& M.R.D.Seaward): 225240. Westbury Press/Linnean Society, London.

Townsend,C.C. 1971.List of Diego Garcia bryophyta. Atoll Research Bulletin 149: 161-162. 